\title{
The mass production and batch test result of the 15k 20-inch MCP-PMT in NNVT for JUNO
}

\author{
Wu Qi ${ }^{a, b}$, Qian Sen ${ }^{a, b^{\star}}$, Cao Yiqi ${ }^{c}$, Chen Pengyu ${ }^{a, b}$, Huang Guorui ${ }^{c}$, Jin Muchun ${ }^{c}$, Jin \\ Zhen $^{c}$, Li Dong ${ }^{c}$, Li Haitao ${ }^{c}$, Li Kun ${ }^{c}$, Liu Shulin ${ }^{a, b}$, Li Zhen ${ }^{c}$, Ma Lishuang ${ }^{a, b}$, Peng \\ Shuo $^{a, b}$, Ren Ling ${ }^{c}$, Si Shuguang ${ }^{c}$, Su Detan ${ }^{c}$, Sun Jianning ${ }^{c}$, Wang Xingchao ${ }^{c}$
}

aInstitute of High Energy Physics, Chinese Academy of Sciences, 100049, Beijing, China

bState Key Laboratory of Particle Detection and Electronics, 100049, Beijing, China

cNorthern Night Vision Technology Co., Ltd., 211100, Nanjing, China

E-mail: qianseihep.ac.cn,

The Jiangmen Underground Neutrino Observatory (JUNO) in China aiming to determine the neutrino mass hierarchy is under construction and the 15,000 MCP-PMTs produced in NNVT, which will be installed as the central liquid scintillator detector of JUNO, have been completed and delivered by August of 2020. During the mass production process, breakthrough of the photocathode technology greatly improved the quantum efficiency (QE) of the MCP-PMT from $30 \%$ to $35 \%$ and so the detection efficiency (DE) has been improved. The performance of other parameters keeps steady during the mass production process of 15,000 MCP-PMTs. The average $\mathrm{QE}$ at $400 \mathrm{~nm}$ for the 15,000 pieces of MCP-PMTs is $32 \%$. With a layer of high-SEY material deposited on the MCP, the collection efficiency can reach nearly $100 \%$ and the average DE at $400 \mathrm{~nm}$ for the $15 \mathrm{k}$ pieces of MCP-PMTs is $31.5 \%$.

40th International Conference on High Energy physics - ICHEP2020

July 28 - August 6, 2020

Prague, Czech Republic (virtual meeting)

*Qian Sen, E-mail: qians@ihep.ac.cn 


\section{Introduction}

The JUNO was proposed with its main purpose of determining the neutrino mass hierarchy using a 20 kton underground liquid scintillator detector and is now under construction in Jiangmen, Guangdong province in south China. In order to capture as much signal light as possible from neutrino reactions in the detection medium, 20,000 pieces of 20-inch photomultiplier tubes (PMTs) will be employed for the Cherenkov light detection, as shown in Figure 1 (left). As a multipurpose underground scintillator observatory, the JUNO have strict requirements on the performance of these PMTs, especially on their QE, DE, sensitive area and peak-to-valley ratio (P/V) of singlephotoelectron spectrum.
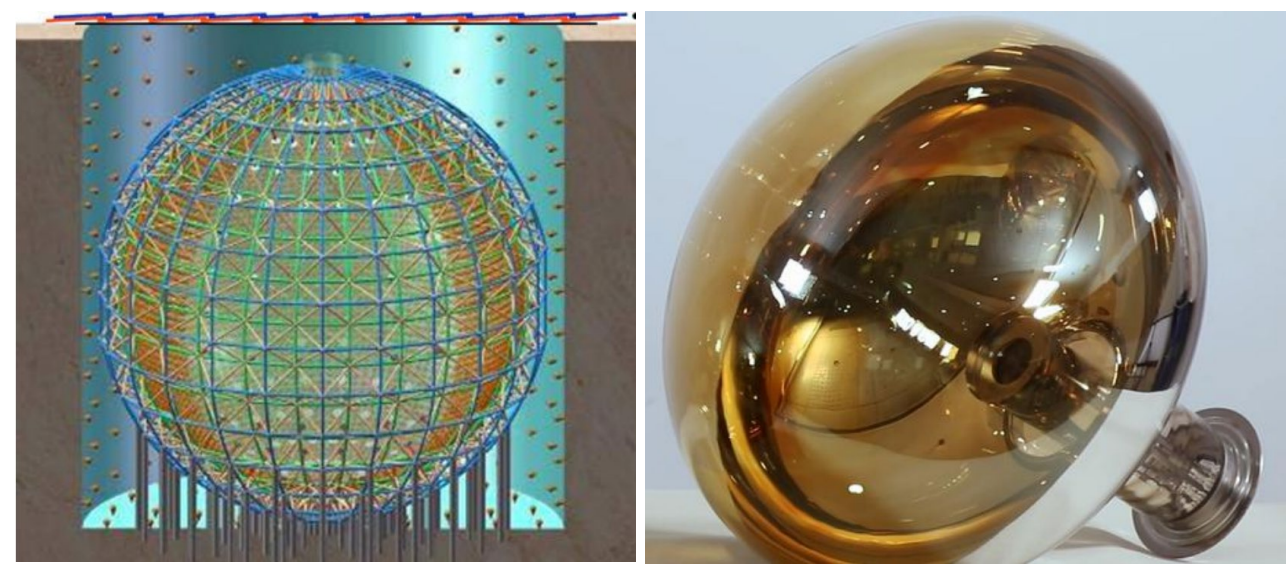

Figure 1: The schematic diagram of the central JUNO detector (left). MCP-PMT produced by NNVT in China (right).

In order to meet the requirements of the JUNO, a new concept of micro-channel plate PMT (MCP-PMT) was conceived by the scientists in the Institute of High Energy Physics (IHEP) in China [1]. The MCP-PMT uses the two-stages MCP as the electron multiplication structure instead of the traditional bulky dynode chains. With the effort of all the members in the MCPPMT workgroup in China, the prototype with high DE of $26 \%$ at $400 \mathrm{~nm}$, which meets the requirements for JUNO, was produced in the Northern Night Vision Technology Co., Ltd. (NNVT) in 2015 [2] and got 75\% of the PMT orders of JUNO (15,000 20-inch MCP-PMTs). Figure 1(right) shows the 20-inch MCP-PMT prototype produced by NNVT. To meet the demands of other neutrino experiments, which require great time performance of the PMTs, the structure of the focusing electrode was optimized and the Flower-Like-MCP-PMT with transit time spread (TTS) less than 5 ns was successfully produced.

The mass production line for the 15,000 MCP-PMTs for JUNO was completed in 2016 and the corresponding batch test system was completed at the beginning of 2017. During the mass production process, the researchers in IHEP and NNVT still made effort to improve the QE of the photocathode and the MCP-PMTs with QE of more than $30 \%$ at $400 \mathrm{~nm}$ were successfully produced in 2017. By August of 2020, all 15k MCP-PMTs are produced and delivered. In this manuscript, the average value for the typical performance parameters of the 15,000 MCP-PMTs is given and the performance change of these MCP-PMTs during mass produciton is analyzed. 


\section{The status of mass production MCP-PMTs for JUNO and latest develepment}

The 20-inch MCP-PMT mass production line was completed in NNVT in 2016 with the capability of producing 30 pieces of tubes per day and the batch test system was completed at the beginning of 2017. All 15k MCP-PMTs for JUNO have been produced, tested and delivered by August of 2020. The MCP-PMT produced by NNVT uses the two-stages MCP as its electronmultiplication structure and the detection efficiency is improved with the use of transmission photocathode on the top hemisphere and the reflective photocathode on the bottom hemisphere [3]. The secondary electron material deposited on the outer surface of the MCP further improves the collection efficiency (CE) into nearly $100 \%$ but it also affects the time performance. For some neutrino experiments such as the Large High Altitude Air Shower Observatory (LHAASO), the Hyper-Kamiokande (HyperK) and the China Jinping Underground Laboratory, PMTs requiring good time performance are needed. Structural optimizations were made and new type of MCP-PMTs were developed to improve the time performance of the MCP-PMTs [4].

In order to improve the time performance of the MCP-PMTs, the structure of the focusing electrode was changed. The flower-like MCP-PMTs use a new structure of focusing electrode with larger area. It can reach a TTS of less than $5 \mathrm{~ns}$ with a dark count of about $20 \mathrm{kHz}$. The LHAASO has already ordered 2270 pieces of 20-inch flower-like-MCP-PMTs and the HyperK PMT group is also testing this kind of MCP-PMT.

\section{PMT test methode}

\subsection{PMT photocathode performance test}

The PMT photocathode performance includes the QE spectral response curve, QE and its corresponding uniformity for the spherical photocathode which can be tested when the light source works in DC mode.

The QE represents the capability of the photocathode to convert photons into electrons. For batch test, the QE is always obtained through relative measurement and the voltage is only applied to the cathode and anode. The first step for QE test is to adjust the voltage between the cathode and the anode under certain light intensity and obtain a steady photocathode output current which means all photoelectrons emitted from the photocathode are collected by the MCP. The photocathode output current of the tested PMT is compared with a reference PMT with known $\mathrm{QE}$ at the same time, and we can obtain the QE of the tested PMT and scale its error using the error of the reference PMT [5].

The material of the photocathode of the PMT determines its ability to convert photons of different wavelengths to electrons, which can be characterized by the QE spectral response curve. The light source which can change its output light wavelength every $10 \mathrm{~nm}$ from $300 \mathrm{~nm}$ to 700 $\mathrm{nm}$ is applied and the $\mathrm{QE}$ at each wavelength is recorded so we can get the spectral response curve.

\subsection{PMT anode performance test}

The PMT anode performance includes the gain, DE, P/V, energy resolution (ER) and TTS which can be tested when the light source works in pulse mode.

When the PMT is working in single-photon-detection mode, the distribution of the area of the tested pulse is called single photoelectron (SPE) spectrum. The SPE spectrum can reflect the anode performance of the MCP-PMT and from the spectrum we can get the value of gain, DE, 
$\mathrm{P} / \mathrm{V}$ ratio, energy resolution and so on. The $\mathrm{P} / \mathrm{V}$ ratio can reflect the ability of MCP-PMT to discriminate single-photoelectron signal.

With the waveform recorded with the oscilloscope, we can obtain the time performance of the MCP-PMT, including the rise time (RT), fall time (FT), transit time (TT), and TTS. TT represents the interval between the light pulse signal and the anode output signal and it obeys Gaussian distribution, the sigma of which represents the time measurement precision and is named TTS [6].

\section{Batch test results}

The 20-inch MCP-PMT mass production line was completed in NNVT in 2016 with the capability of producing 30 pieces of tubes per day and the batch test system was completed at the beginning of 2017. All 15k MCP-PMTs for JUNO have been produced, tested and delivered by August of 2020.

Table 1 shows the performance of the MCP-PMT prototypes in 2015 and the average values of performance parameters of MCP-PMTs mass produced for JUNO between 2017 and 2020 [7]. The average QE at $400 \mathrm{~nm}$ is $32.0 \%$ and the dark rate is $40 \mathrm{kHz}$ for the $15 \mathrm{k}$ MCP-PMTs.

\begin{tabular}{cccccc}
\hline Parameters & unit & Prototypes & 1k pieces & 10k pieces & 15k pieces \\
\hline QE@400 nm & $\%$ & 26.0 & 29.3 & 31.3 & 32.0 \\
DE@400 nm & $\%$ & 26.0 & 29.2 & 30.9 & 31.5 \\
ER & $\%$ & 41.0 & 33.1 & 31.9 & 32.1 \\
P/V ratio & & 5.6 & 7.1 & 7.1 & 7.1 \\
Dark Rate & $\mathrm{kHz}$ & 30.0 & 36.9 & 37.8 & 40.0 \\
Rise Time & $\mathrm{ns}$ & 1.2 & 1.4 & 1.44 & 1.4 \\
Fall Time & $\mathrm{ns}$ & 10.2 & 25.2 & 23.5 & 24.0 \\
TTS & $\mathrm{ns}$ & 12.0 & 20.0 & 20.0 & 20.0 \\
High Voltage & $\mathrm{V}$ & 1930.0 & 1767.0 & 1745.0 & 1730.0 \\
\hline
\end{tabular}

Tabel 1: The average values of performance parameters of the MCP-PMTs for JUNO.

\subsection{SPE detection performance}

The PMT parameters ER and P/V are obtained from the SPE spectrum. ER can represent the ability to distinguish between different energy peaks and $\mathrm{P} / \mathrm{V}$ can represent the ability to realize single-photon detection. Both of them are stable during the production process, as can be seen from Figure 2, and the average value for ER and $\mathrm{P} / \mathrm{V}$ is $32.1 \%$ and 7.1 , respectively.
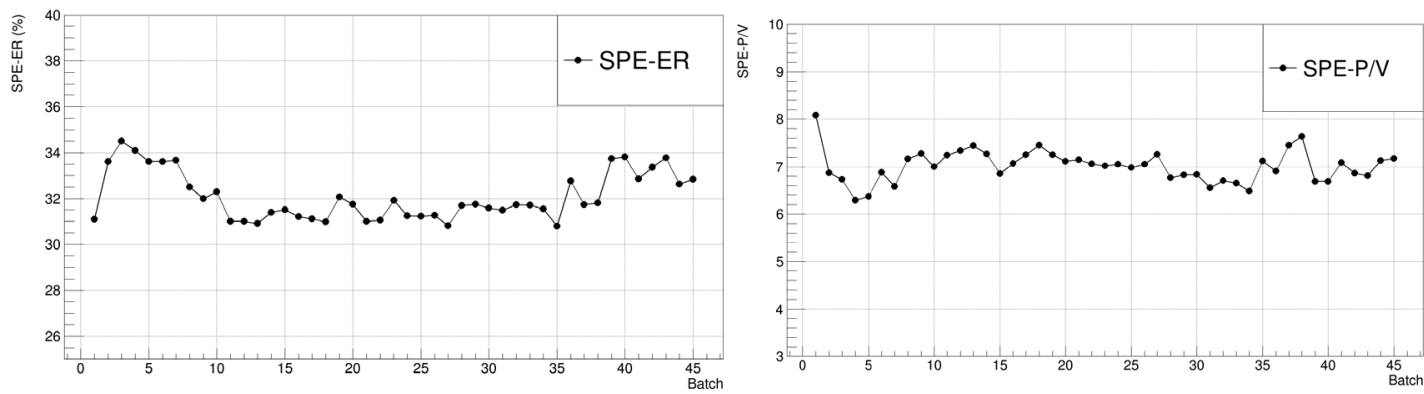

Figure 2: The ER (left) and P/V (right) changes with different batches. 


\subsection{QE performance}

The photocathode (PC) of the MCP-PMTs developed for JUNO is Bi-alkali PC, whose standard QE is about $25 \%$ at $400 \mathrm{~nm}$. The tested QE can reflect the performance of the photocathode and since the CE is nearly $100 \%$, the trend of DE is consistent with the QE. After the technological breakthrough of photocathode, the average QE could reach above $32 \%$ at Imathrm $\{400 \backslash \mathrm{nm}\}$ and all the PMTs for JUNO are of high QE since June 2018. These MCPPMTs with high QE are called HQE-MCP-PMT. The CE was also improved as shown in Figure 3 [8]. The photocathode of the HQE-MCP-PMT is also Bi-alkali PC, with the peak wavelength changed from $390 \mathrm{~nm}$ to $380 \mathrm{~nm}$ and for its high QE it is named Super-Bi-alkali (SBA).
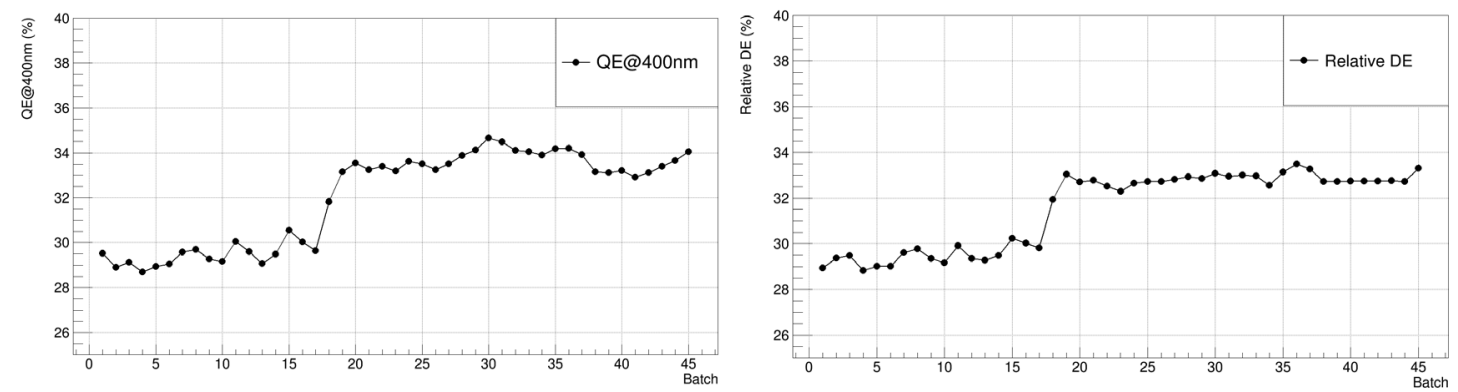

Figure 3: The QE (left) and DE (right) changes with different batches.

\subsection{Dark rate performance}

The dark noise of PMT is caused by lots of factors, including the thermal electron emission of the photocathode, the weak radiation from the tube, the residual gases in the vacuum tube and the temperature of the working environment. The dark rate (DR) of the 15k MCP-PMTs is relatively steady and the average value is $40 \mathrm{kHz}$ as we can see from Figure 4.

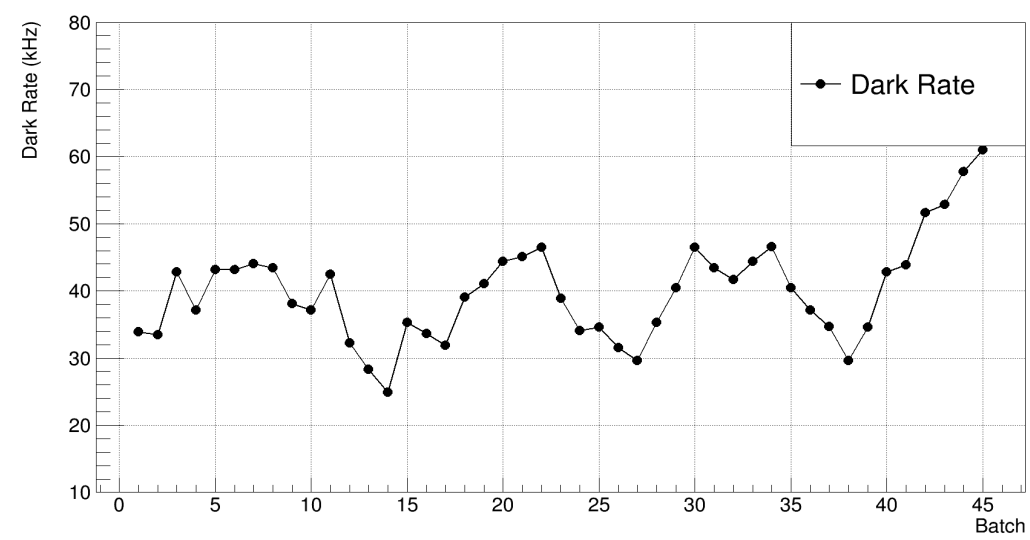

Figure 4: The Dark Rate changes with different batches.

\subsection{Time performance}

The time performance of PMTs is determined by its structure and the test methode. As we can see from Figure 5, the RT and TTS remain steady during the mass production process without the structure change of MCP-PMT. The average value of TTS is about $20 \mathrm{~ns}$. 

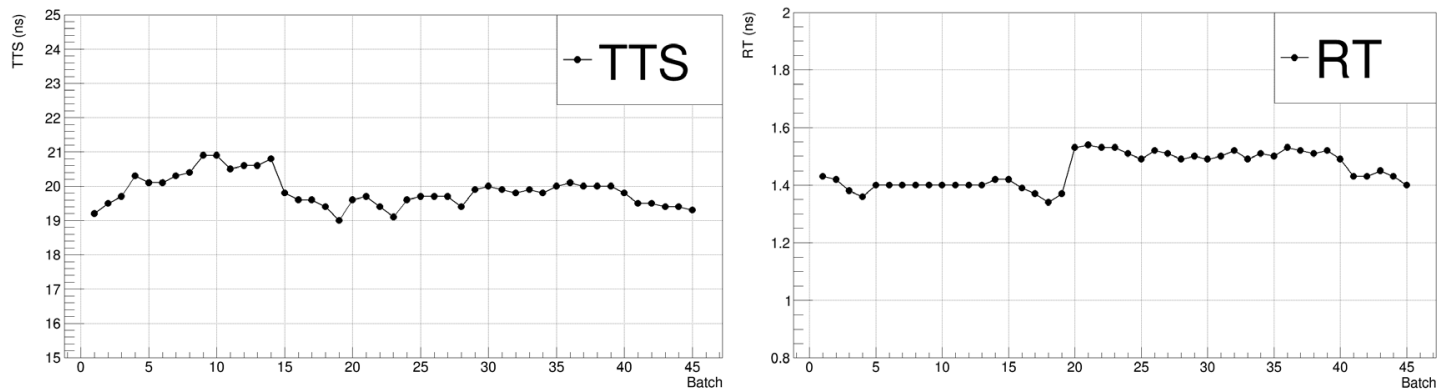

Figure 5: The TTS and RT changes with different batches.

\section{Conclusions}

The 15,000 MCP-PMTs for JUNO have been produced, tested and delivered by August of 2020 in NNVT. Due to the breakthrough of the photocathode production technology, the QE of these MCP-PMTs was improved greatly in 2018 from $30 \%$ to $35 \%$ and the DE was also improved. Other parameters, such as the DR, TTS, gain, P/V ratio, keep steady during the process of mass production.

\section{Acknowledgement}

This project has been partially supported by the National Natural Science Foundation of China (No.11675205), Foundation of State Key Laboratory of Particle Detection and Electronics (SKLPDE-ZZ201902), Program of Science \&Technology Service Network of CAS(KFJ-STS-QYZX-050), and the Youth Innovation Promotion Association CAS (YIPA2017017).

\section{References}

[1] Y. F. Wang et al., A new design of large area MCP-PMT for the next generation neutrino experiment. NIMA, vol. 695, pp. 113-117, Dec 112012.

[2] Feng Gao et al., Status of the 20 inch MCP-PMT prototype development for JUNO experiment, 1 2017 J. Phys.: Conf. Ser. 888012050.

[3] Gao F, Qian S et al., The Status of the Batch Test of 20 inch MCP-PMT. In Proceedings of International Conference on Technology and Instrumentation in Particle Physics, 233-236 (Springer Singapore, Singapore, 2018).

[4] Qi Wu et al., The Design of large area MCP-PMT for Neutrino Detector, Nineteenth Lomonosov Conference on Elementary Particle Physics.

[5] Feng Gao, Sen Qian et al., The Large PMT Quantum Efficiency Test Platform, Proceedings of International Conference on Technology and Instrumentation in Particle Physics 2017 pp 304-308.

[6] Feng Gao, Sen Qian et al., Data acquisition system for single photoelectron spectra of photoelectric detector, NUCLEAR TECHNIQUES, Vol.42, No.10, October 2019.

[7] Sen Q et al., Status of the large area MCP-PMT in China, Proceedings of the 38th International Conference on High Energy Physics (ICHEP2016). 3-10 August 2016. Chicago, USA.

[8] Qi Wu et al., The Status of the 20 inch MCP-PMT and its APR Test Result, 2019 IEEE Nuclear Science Symposium and Medical Imaging Conference (NSS/MIC), Manchester, United Kingdom, 2019, pp. 1-4. 